REVISTA DE GEOCIÊNCIAS DO NORDESTE

Northeast Geosciences Journal

v. $7, \mathrm{n}^{\mathrm{o}} 1(2021)$

https://doi.org/10.21680/2447-3359.2021v7n1ID19859

ISSN: 2447-3359

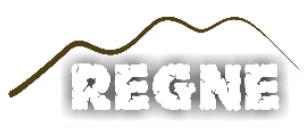

\title{
LEVANTAMENTO DA MACROFAUA EDÁFICA EM ÁREAS DE PROTEÇÃO AMBIENTAL NO MARANHÃO
}

\begin{abstract}
Alana Ellen de Sousa Martins ${ }^{1}$; Judson Chaves
Rodrigues $^{2}$; Maira Rebeca de Alencar Costa

Silva $^{3}$; Maria Tereza Alves Souza ${ }^{4}$; Francilene

Oliveira Lima ${ }^{5}$; Márcia Verônica Pereira

Gonçalves $^{6}$; Rita de Kássia dos Santos Barros ${ }^{7}$; Luiza Daiana Araújo da Silva Formiga ${ }^{8}$
\end{abstract}

${ }^{1}$ Graduada em Ciências Biológicas, Departamento de Química e Biologia, Universidade Estadual do Maranhão (UEMA), Caxias/MA, Brasil.

ORCID: https://orcid.org/0000-0002-3543-8972

Email: a.lanasousa2009@hotmail.com

${ }^{2}$ Graduando em Ciências Biológicas, Departamento de Química e Biologia, Universidade Estadual do Maranhão (UEMA), Caxias/MA, Brasil.

ORCID: https://orcid.org/0000-0001-9236-2508

Email: judsoom.rodriguesz@gmail.com

${ }^{3}$ Graduanda em Ciências Biológicas, Departamento de Química e Biologia, Universidade Estadual do Maranhão (UEMA), Caxias/MA, Brasil.

ORCID: $\underline{\text { https://orcid.org/0000-0002-0648-8022 }}$

Email: mairarebeca07@gmail.com

${ }^{4}$ Graduanda em Ciências Biológicas, Departamento de Química e Biologia, Universidade Estadual do Maranhão (UEMA), Caxias/MA, Brasil.

ORCID: https://orcid.org/0000-0002-8411-5387

Email: mari.tereza02@gmail.com

${ }^{5}$ Graduada em Ciências Biológicas, Departamento de Química e Biologia, Universidade Estadual do Maranhão (UEMA), Caxias/MA, Brasil.

ORCID: $\underline{\text { https://orcid.org/0000-0001-9256-2462 }}$

Email: fran.oliveira353@gmail.com

${ }^{6}$ Graduada em Ciências Biológicas, Departamento de Química e Biologia, Universidade Estadual do Maranhão (UEMA), Caxias/MA, Brasil.

ORCID: https://orcid.org/0000-0001-7805-1463

Email: mv186343@gmail.com
${ }^{7}$ Graduada em Ciências Biológicas, Departamento de Química e Biologia, Universidade Estadual do Maranhão (UEMA), Caxias/MA, Brasil.

ORCID: https://orcid.org/0000-0002-5307-8528

Email: kssyabarros@hotmail.com

${ }^{8}$ Doutora em Zootecnia, Centro de estudos Supeiores de Caxias, Universidade Estadual do Maranhão (UEMA), Caxias/MA, Brasil.

ORCID: https://orcid.org/0000-0001-5001-3297

Email: luidadaiana@hotmail.com

\section{Resumo}

O estudo da estrutura da comunidade da macrofauna edáfica é um meio de entender o funcionamento do solo e os possíveis bioindicadores de suaqualidade. Neste contexto, objetivou-se realizar um levantamento da biodiversidade da macrofauna do solo em duas áreas de Proteção Ambiental no Maranhão. As áreas experimentais consideradas no estudo foram: Área I (APA do Buriti do Meio) e Área II (APA do Inhamum). Para realização das coletas da macrofauna do solo utilizou-se armadilhas do tipo Provid. Foi realizada uma análise faunística, e calculados os índice de diversidade, equabilidade e riqueza das ordens no programa ANAFAU. No geral, foram contabilizados 6.695 indivíduos, distribuídos em 15 ordens. Aordem que mais se destacou dentre as duas áreas foi Hymenoptera, considerada superdominante, superabundante, superfrequente e constante. A precipitação puvial pode interferer diretamente na abundância de indivíduos da macrofauna edáfica. O ambiente da Área II apresentou maior riqueza, diversidade e equabilidade. $\mathrm{O}$ trabalho relevou que as práticas de manejo de agricultura interferem no comportamento da fauna do solo, indicando que a mesma é mais abundante em ambientes mais conservados, confirmando que osorganismos edáficos são bons bioindicadores da qualidade do solo.

Palavras-chave: Abundância; Diversidade; Insetos do solo.

\section{SURVEY OF MACROFAUA EDÁFICA IN PROTECTED AREAS IN MARANHÃO}

\section{Abstract}

Analysis of soil macrofaunal community structure can contribute to our understanding of soil dynamics and bioindicators. This study aimed to survey the biodiversity of soil macrofauna in two 
Environmental Protection Areas (EPA) in Maranhão State, Brazil. Experimental sites were as follows: site I, Buriti do Meio EPA; and site II, Inhamum EPA. Provid-type traps were used to sample soil macrofaunal individuals. A faunistic analysis was carried out, and diversity, evenness, and richness indices of orders were calculated using ANAFAU software. Overall, 6,695 individuals were collected, distributed in 15 orders. Hymenoptera was the most prominent order at both sites, being considered superdominant, superabundant, superfrequent, and constant. Rainfall may directly interfere with the abundance of soil macrofaunal individuals. Site II showed greater richness, diversity, and evenness. This study revealed that agricultural management practices interfere with the behavior of soil macrofauna, showing that soil organisms are more abundant in more preserved environments and may therefore serve as bioindicators of soil quality.

Keywords: Abundance; Diversity; Soil insects.

\section{RELEVAMIENTO DEL MACROFAUA EDÁFICA EN ÁREAS PROTEGIDAS EN MARANHÃO}

\section{Resumen}

El estudio de la estructura comunitaria de la macrofauna edáfica es un medio para comprender el funcionamiento del suelo y los posibles bioindicadores de su calidad. En este contexto, el objetivo fue realizar un estudio de la biodiversidad de la macrofauna del suelo en dos áreas de Protección enMaranhão. Las áreas experimentales consideradas en el estudio fueron Área I (APA de Buriti do Meio) y Área II (APA de Inhamum). Se utilizaron trampas providas para recolectar la macrofauna del suelo. Se realizó análisis de fauna y se calculó el índoce de diversidad, equidad y riqueza de los pedidos en el programa ANAFAU. En total, se registraron 6.695 personas, distribuidas en 15 pedidos. El orden que más se destacó entre las dos áreas fue el orden Hymenoptera, considerado superdominante, superabundante, superfrecuente y constante. Las precipitaciones pueden interferir directamente con la abundancia de individuos en la macrofauna edáfica. El entorno del Área II mostró mayor riqueza, diversidad y equidad. El trabajo reveló que las prácticas de manejo agrícola interfieren en el comportamiento de la fauna del suelo, indicando que es más abundante en ambientes más conservados, confirmando que los organismos edáficos son buenos bioindicadores de la calidad del suelo.

Palabras-clave: Abundancia; Diversidad; Insectos del suelo.

\section{INTRODUÇÃO}

O solo faz parte do processo de produção da grande maioria dos produtos consumidos, pois é a partir dele que é retirado praticamente todos os alimentos. Além disso, é um ambiente natural no qual muitos organismos se desenvolvem gerando importantes benefícios aos seres humanos (RENNER et al., 2017) e também aos outros organismos vivos presentes no solo ou que fazem uso dele. A biodiversidade do Brasil é uma das maiores do planeta, sendo a fauna do solo um importante componente dessa diversidade (MELO et al., 2009). O solo caracteriza-se por ser um reservatório faunístico composto de uma grande diversidade de organismos que garantem o biofuncionamento e a sustentação de todo o bioma (JACOBS et al., 2007), uma vez que a maioria dos nutrientes disponíveis no solo para o crescimento das plantas depende de complexas interações entre raízes, microrganismos e a fauna edáfica (GESTEL et al., 2003).

A fauna do solo é dividida em três grupos: microfauna, mesofauna e macrofauna. Esses organismos trabalham em conjunto, preservando a integridade física, química e biológica do solo, contribuem para a ciclagem de ciclagem de nutrientes, fazem trocas gasosas entre a atmosfera e o solo, além de outros benefícios ao solo e as plantas (RENNER et al., 2017). A macrofauna é influenciada tanto pela quantidade, quanto pela qualidade do material vegetal presente no solo. Além disso, esses organismos indicam as condições ecológicas dos ambientes edáficos (CARRILLO et al., 2011). O estudo da estrutura da comunidade da macrofauna edáfica é um meio de entender o funcionamento do solo e os possíveis bioindicadores de sua qualidade (AQUINO et al., 2008). Organismos indicadores de qualidade são grupos ou comunidades cuja presença, ausência, densidade, diversidade e papel funcional revelam a possibilidade de uma situação adversa provocada naturalmente ou por algum fator antrópico (BARETTA et al., 2010).

A macrofauna do solo é formada por todos os invertebrados (animais sem coluna vertebral), maiores que $2 \mathrm{~mm}$ e que vivem no solo em pelo menos uma fase do seu ciclo de vida, seja na fase adulta ou na imatura. Devido seu tamanho, podemos visualizá-los a olho nu, ou seja, sem a necessidade de lupa ou microscópio. A macrofaunainclui organismos de diferentes classes, ordens e famílias de invertebrados, sendo representada por mais de 20 grupos taxonômicos. Na macrofauna invertebrada do solo estão os organismos mais conhecidos pelos agricultores e mais estudados pelos pesquisadores, conhecidos popularmente como as minhocas, formigas, cupins, besouros, centopeias, piolhos-decobra, baratas, tesourinhas, grilos, gafanhotos, caracóis, percevejos, tatuzinhos, cigarras, lagartas, aranhas, opiliões, pseudoescorpiões, entre outros (KORASAKI et al., 2017).

No solo, as principais atividades desses organismos são: decomposição da matéria orgânica, produção de húmus, ciclagem de nutrientes e energia, , dentre outros (HOFFMAN et al., 2009). A maior concentração dos organismos pertencentes à macrofauna encontra-se na camada superficial de $0-10 \mathrm{~cm}$ de profundidade. Esta é a camada mais afetada pelas práticas de manejo como preparo do solo, adubação e decomposição de resíduos orgânicos (BARETTA et., 2006).

As áreas da pesquisa estão localizadas dentro do bioma Cerrado, no estado do Maranhão, e compreendem a, Área de Proteção Ambiental Municipal do Inhamum (APA do Inhamum) e a Área de Proteção Ambiental Buriti do Meio.Ambas apresentam determinadas atividades antrópicas, como desmatamentos e, principalmente, queimadas, que podem ter efeitos catastróficos, comprometendo a integridade da fauna e flora das APA's. Perante desses impactos sofridos, trabalhos evidenciando os impactos ambientais causados pelo homem na APA do Inhamum e na APA do Buriti do Meio, têm como finalidade sensibilizar e promover a conscientização da população circunvizinha. Além disso, esse tipo de trabalho promove embasamento e comprovação dos impactos, para fins de políticas públicas voltadas para área de conservação.

Diante do exposto, o presente trabalho tem o objetivo de realizar um levantamento da biodiversidade da macrofauna do 
solo em duas áreas de Proteção Ambiental no Maranhão.

\section{METODOLOGIA}

A pesquisa foi realizada em duas áreas de proteção ambiental localizadas no município de Caxias-MA. A área denominada como Área I é a Área de Proteção Ambiental Municipal do Buriti do Meio, localizada no Projeto de Assentamento do Buriti do Meio e Santa Rosa, no $2^{\circ}$ Distrito. Esta área dista $35 \mathrm{~km}$ do perímetro urbano da cidade de Caxias, e é caracterizada por apresentar vegetação secundária, advindas de práticas de manejo de agricultura, além de ser frequentemente desmatada e queimada. O clima da região é tipo subúmido seco, com temperatura média anual de $26^{\circ} \mathrm{C}$ e $27^{\circ} \mathrm{C}$ e precipitação pluvial entre 1.600 a $2.000 \mathrm{~mm}$, com período seco de junho a novembro, e chuvoso de dezembro a maio (MONTES et al., 1997).

A área denominada como Área II, é a Área de Preservação Ambiental Municipal do Inhamum, localizada à margem esquerda da BR-316, próximo ao perímetro urbano de Caxias. Esta área caracteriza-se por apresentar uma vegetação típica de Cerrado, que vai desde campo limpo até cerradão. Devido seu posicionamento entre o Nordeste Semiárido e o Meio-Norte, essa região possui um clima equatorial quente e úmido, com duas estações distintas: verõeschuvosos e invernos secos, com temperatura média anual superior a $24^{\circ} \mathrm{C}$ e índices pluviométricos regulares entre 1.600 e $1.800 \mathrm{~mm}$, (ARAÚJO, 2012).

Em cada área foram delimitados quatro transectos paralelos, distando aproximadamente $10 \mathrm{~m}$ entre si. Em cada transecto, foram demarcadas cinco unidades amostrais equidistantes $(10 \mathrm{x}$ $10 \mathrm{~m}$ ), de modo que foram amostrados 20 pontos em cada tratamento (áreas), totalizando 40 pontos amostrais.

Para a captura da macrofauna do solo, foram utilizadas armadilhas de queda do tipo Provid. A armadinha é constituída por uma garrafa PET com capacidade de $2 \mathrm{~L}$, com quatro orifícios com dimensões de $2 \times 2 \mathrm{~cm}$ (ARAUJO, 2010) na altura de $20 \mathrm{~cm}$ de sua base, contendo $200 \mathrm{~mL}$ de uma solução de detergente a uma concentração de 5\%, e adicionadas 5 gotas de Formol P.A. (Formaldeido). As armadilhas foram enterradas com os orifícios ao nível da superfície do solo e foram mantidas no mesmo local em todas as coletas, permanecendo no campo por um período de quatro dias (96 horas). Foram realizadas quatro coletas: duas no período seco (setembro e outubro/2018) e duas no período chuvoso (fevereiro e março/2019), contemplando as das áreas de estudo.

Após o período de 96 horas, as armadilhas foram retiradas do campo e identificadas de acordo com data da coleta, área e ponto. Em seguida foram transportadas para o laboratório onde os conteúdos das armadilhas foram devidamente lavados em água corrente, com auxílio de peneira de $0,25 \mathrm{~mm}$ e transferidos para potes plásticos contendo álcool etílico a 70\%. Posteriormente, com o auxílio de lupa, pinças e placas de petri, foi feita a contagem e identificação dos organimos de acordo com a classe, ordem e/ou grupo taxonômico, utilizando a chave de identificação proposta por Triplehorn e Jonnson (2011).

Os dados dos invertebrados da macrofauna do solo foram submetidos a análise faunística com base nos índices de frequência, constância e dominância selecionando as ordens predominantes, ou seja, aquelas que apresentam maiores índices faunísticos (SILVEIRA NETO et al., 1976). Foi realizado também análises dos índices de diversidade de Shannon - Weaner (H'), equabilidade de Pielou (e) e riqueza de Margalef, que foram feitas no programa ANAFAU (MORAES et al., 2003).

A frequência (f) das ordens taxonômicas foi determinada pela participação percentual do número de indivíduos de cada ordem, em relaçãoao total coletado. A partir dos resultados que obtidos foram estabelecidas as classes de frequências para as ordens de acordo com intervalo de confiança (IC) da média aritmética a 5\% de probabilidade (SILVEIRA NETO et al., 1976). As classes foram obtidas através da aplicação dos seguintes critérios: Pouco frequente $(\mathrm{PF})$ - frequência menor que olimite inferior do IC a $5 \%$; Frequente $(\mathrm{F})$ - frequência situada dentro do IC a 5\%; e Muito frequente (MF) - frequência maior que olimite superior do IC a $5 \%$.

A constância foi calculada por meio da porcentagem de ocorrência das ordens no levantamento, de acordo com os percentuais obtidos, as ordens taxonômicas foram separadas em categorias segundo a classificação de Bodenheiner (1955) citado por Silveira Neto et al. (1976): Ordens Constantes (W) = presentes em mais de 50\% das coletas; Espécies Acessórias $(\mathrm{Y})=$ presentes em 20 - 50\% das coletas; Espécies Acidentais $(\mathrm{X})=$ presentes em menos de $20 \%$ das coletas.

A dominância consiste na capacidade da ordem modificar, em benefício próprio, o impacto recebido do ambiente, podendo causar o aparecimento ou desaparecimento de outros organismos (SILVEIRA NETO et al., 1976). A partir dos valores obtidos, as ordens foram classificadas em: Dominante (D) - quando os valores da frequência foram superiores ao índice de dominância; e Não Dominante (ND) - quando os valores da frequência foram inferiores ao índice de dominância.

Também foi verificada a temperatura do solo a $10 \mathrm{~cm}$ de profundidade, em cada ponto de coleta, com a utilização de termômetro digital do tipo espeto, modelo: K29-5030, marca: Kasvi. Além disso, a precipitação pluvial das duas áreas, são as mesmas por estarem na mesma cidade (mesma estação para ambas as áreas), e foram fornecidas mensalmente pela Estação Meteorológica de Caxias.

\section{RESULTADOS E DISCUSSÃO}

\subsection{Abundância, frequência, constância e dominância dos grupos taxonômicos}

Verificou-se que os indivíduos que compõe a macrofauna invertebrada do solo apresentaram variações entre as duas áreas experimentais. No geral, foram contabilizados 6.695 indivíduos, sendo 3.496 na Área I (APA do Buriti do Meio), e 3.199 na Área II (APA do Inhamum), distribuídos em 15 ordens (Tabela 1).

$\mathrm{Na}$ Área I, em ordem decrescente quanto ao número de indivíduos, a ordem Hymenoptera apresentou percentual de indivíduos e , foi considerada superdominante, superabundante, superfrequente e constante, seguida da ordem Coleoptera também considerada superabundante, superfrequente e constante. A ordem Diptera com 159 indivíduos, foi considerada dominante, muito abundante, muito frequente e constante, seguida da ordem Orthopera com 136 indivíduos foi considerada dominante, muito abundante, muito frequente e acessória e Araneae com 106 indivíduos foi considerada dominante, muito abundante muito frequente e acessória. 
As ordens Embioptera, Hemiptera e Scorpiones foram exclusivas para a Área I (Tabela 1). Os indivíduos da ordem Embioptera, são encontrados na terra (até $30 \mathrm{~cm}$ de profundidade) sob troncos, sob pedras, na base de árvores, arbustos, cactáceas, na casca de árvores, independentemente do ambiente (ROSS, 2000). Diante disso, não houve um fator determinante para o aparecimento de tais indivíduos nesta área. Já no caso dos indivíduos da ordem Hemiptera, a exclusividade se deve ao fato de na área haver plantações, e alguns representantes da ordem estão intimamente relacionados à estes ambientes. Isso ocorre por eles apresentarem preferência por zonas de crescimento do vegetal, como as brotações, folhas novas e botões florais, onde os mesmos sugam continuamente a seiva das plantas, provocando amarelecimento, enrugamento, deformação e definhamento, podendo causar a morte pelo enfraquecimento generalizado (RAFAEL et al., 2012). A ordem
Scorpiones também foi exclusiva para Área I. A grande maioria das espécies de escorpiões apresenta exigências específicas com relação ao hábitat e micro-hábitat e possuem padrões ecológicos e biogeográficos previsíveis e localizados (LOURENÇO; EICKSTEDT, 2009). No entanto, ainda de acordo com os mesmos autores, algumas espécies do gênero Centruroides, Isometrus, Tityus, Euscorpius e Bothriurus apresentam alta plasticidade ecológica e padrões irregulares de distribuição, podendo ocorrer inclusive em ambientes perturbados ou modificados pela ação do homem.

$\mathrm{Na}$ Área II em ordem decrescente quanto ao número de indivíduos, a ordem Hymenoptera com 2.523 indivíduos, , também foi considerada superdominante, superabundante, superfrequente e constante, seguida da ordem Coleoptera com 314 indivíduos, Diptera com 195 indivíduos e Araneae com 99 indivíduos, também consideradas superdominante, superabundante, superfrequente e constante. A ordem Orthopera com 16 indivíduos foi considerada dominante, abundante, muito frequente e acessória.

Tabela 1. Relação dos grupos de indivíduos da macrofauna encontradosnaÁrea I (APA do Buriti do Meio) e Área II (APA do Inhamum) Caxias, Maranhão. Fonte: Autor (2019).

\begin{tabular}{|c|c|c|c|c|c|c|c|c|c|c|c|c|c|c|c|c|}
\hline \multirow[b]{2}{*}{ Filo } & \multirow[b]{2}{*}{ Classes } & \multirow[b]{2}{*}{ Ordens } & \multicolumn{7}{|c|}{ Área I } & \multicolumn{7}{|c|}{ Área II } \\
\hline & & & NI & $\%$ & $\mathrm{NC}$ & D & $\mathbf{A}$ & $\mathbf{F}$ & $\mathrm{C}$ & NI & $\%$ & $\mathrm{NC}$ & D & $\mathbf{A}$ & $\mathbf{F}$ & $\mathbf{C}$ \\
\hline \multirow{16}{*}{ Arthropoda } & Arachnida & Araneae & 106 & 3,03 & 53 & $\mathrm{D}$ & $\mathrm{ma}$ & $\mathrm{MF}$ & $\mathrm{W}$ & 99 & 3,09 & 46 & SD & sa & $\mathrm{SF}$ & $\mathrm{W}$ \\
\hline & \multirow{9}{*}{ Insecta } & Archeognatha & - & - & - & - & - & - & - & 1 & 0,03 & 1 & ND & d & PF & $\mathrm{Z}$ \\
\hline & & Blattária & 13 & 0,37 & 12 & $\mathrm{D}$ & $\mathrm{c}$ & F & $\mathrm{Y}$ & 26 & 0,81 & 15 & $\mathrm{D}$ & ma & MF & $\mathrm{Y}$ \\
\hline & & Coleoptera & 590 & 16,88 & 50 & SD & sa & SF & W & 314 & 9,82 & 45 & SD & sa & SF & W \\
\hline & & Diptera & 159 & 4,55 & 46 & D & ma & MF & W & 195 & 6,10 & 47 & SD & sa & SF & $\mathrm{W}$ \\
\hline & & Embioptera & 1 & 0,03 & 1 & ND & d & PF & $\mathrm{Z}$ & - & - & - & - & - & - & - \\
\hline & & Hemiptera & 9 & 0,26 & 8 & $\mathrm{D}$ & $\mathrm{c}$ & $\mathrm{F}$ & $\mathrm{Z}$ & - & - & - & - & - & - & - \\
\hline & & Hymenoptera & 2.472 & 70,71 & 74 & SD & sa & SF & W & 2523 & 78,87 & 67 & SD & sa & SF & $\mathrm{W}$ \\
\hline & & Isoptera & - & - & - & - & - & - & - & 2 & 0,06 & 2 & ND & $\mathrm{c}$ & $\mathrm{F}$ & $\mathrm{Z}$ \\
\hline & & Lepidoptera & 1 & 0,03 & 1 & ND & d & $\mathrm{PF}$ & $\mathrm{Z}$ & 1 & 0,03 & 1 & ND & d & $\mathrm{PF}$ & $\mathrm{Z}$ \\
\hline & Chilopoda & Lethobiomorpha & - & - & - & - & - & - & - & 1 & 0,03 & 1 & ND & d & PF & $\mathrm{Z}$ \\
\hline & Insecta & Orthoptera & 136 & 3,89 & 40 & $\mathrm{D}$ & $\mathrm{ma}$ & MF & $\mathrm{Y}$ & 16 & 0,50 & 8 & $\mathrm{D}$ & $\mathrm{a}$ & MF & $\mathrm{Y}$ \\
\hline & \multirow{2}{*}{ Arachnida } & Pseudoescorpiones & 6 & 0,17 & 6 & $\mathrm{D}$ & $\mathrm{c}$ & $\mathrm{F}$ & $\mathrm{Z}$ & 20 & 0,63 & 15 & $\mathrm{D}$ & $\mathrm{ma}$ & $\mathrm{MF}$ & $\mathrm{Y}$ \\
\hline & & Scorpiones & 3 & 0,09 & 2 & ND & d & $\mathrm{PF}$ & $\mathrm{Z}$ & - & - & - & - & - & - & - \\
\hline & \multirow[t]{2}{*}{ Chilopoda } & Scutgeromorpha & - & - & - & - & - & - & - & 1 & 0,03 & 1 & ND & d & $\mathrm{PF}$ & $\mathrm{Z}$ \\
\hline & & Total & 3496 & 100 & & & & & & 3199 & 100 & & & & & \\
\hline
\end{tabular}

Programa ANAFAU: NI= número de indivíduos; $N C=$ número de coletas; $D=$ Dominância $-($ sd $)$ super dominante; $(d)$ dominante; (nd) nãodominante. A= Abundância - (sa) super abundante; (ma) muitoabundante; (c) comum; (d) dispersa. F= Frequência - (SF) super frefrequente; $(M F)$ muito frequente; $(F)$ frequente; $(P F)$ pouco frequente. $C=$ Constância $-(W)$ constante; $(Y)$ acessória; $(Z)$ acidental.

As ordens Archeognatha, Isoptera, Lethobiomorpha e Scutigeromorpha foram exclusivas para a Área II (Tabela 1). Os indivíduos das ordens Archeognatha, Lethobiomorpha e Scutigeromorpha, preferem ambientes úmidos e são encontrados sob a casca de árvores no solo, ocupando serrapilheiras e troncos em estágio de decomposição (RAFAEL et al., 2012; GREGORY; GIRIBERT, 2007). O fato desses organimos serem exclusivos para estaárea, pode ser explicado por isso, pois a mesma dispõe de um ambiente com grande quantidade de serrapilheira, material orgânico em decomposição e cascas de árvores, o que pode propiciar o aparecimento de indivíduos dessas ordens. A presença de indivíduos da ordem Isoptera para esta área, em parte também está relacionado com as mesmas características citadas para o aparecimento das outras ordens exclusivas, no entanto, necessariamente o fato da existência de muita serapilheira pode explicar explica a ocorrência dessa ordem. Materiais orgânicos servem de alimento para a fauna edáfica, e, consequentemente, maior estabilidade para os diversos organismos. Favorece, ainda, 
a presença de predadores mais ativos, uma vez que algumas espécies de minhocas, formigas e cupins são mais generalistas e necessitam basicamente de boa estrutura do solo (do ponto de vista físico) (ROSA et al., 2015).

De forma geral, houve predominância da ordem Hymenoptera, em relação aos demais grupos encontrados. A literatura aponta que muitos trabalhos com macrofauna edáfica em áreas de Cerrado e utilizando o mesmo método de coleta, trazem resultados semelhantes aos encontrados neste trabalho (BUSSINGUER, 2018;; ARAÚJO et al., 2010). Outros trabalhos em diferentes biomas como Caatinga (SANTOS et al., 2018; SANTOS, 2014) e Mata Atlântica (MACHADO et al., 2015) por exemplo, também mostram que o grupo Hymenoptera é o mais dominante. Tal acontecimento nesta área pode estar relacionado com a predominância dos indivíduos da família Formicidae. Os formicídeos são um grupo considerado de altariqueza de espécies, por possuírem táxons especializados, pela grande sensibilidade em perceber alterações ambientais e serem relativamente fáceis de coletar (SOUZA et al., 2018).

Os indivíduos da ordem Coleoptera se fizeram presentes nas duas áreas, porém houve maior ocorrência de indivíduos na Área I. Esse resultado pode estar relacionado ao ambiente que a área proporciona para esses indivíduos que é uma área onde é realizado práticas de lavoura, como o plantio de culturas como feijão, milho, mandioca, dentre outros. $\mathrm{O}$ fato das armadilhas estarem diretamente no local que se encontra a plantação, favorece o aparecimento de alguns besouros específicos, que podem está se beneficiando do ambiente, como é o caso dos adultos de Meloidae, que segundo Marinone e Ganho (2003), são desfolhadores e considerados como pragas de plantas cultivadas. Por outro lado, a ordem Coleoptera é a maior ordem dentro da classe Insecta, abrigando uma enorme quantidade de espécies que ocupam os mais diversos nichos ecológicos,onde no grupo está incluso grande número de espécies consideradas pragas agrícolas e que apresentam grande diversidade de hábitos alimentares (AUDINO et al., 2007).

A maior ocorrência de indivíduos da ordem Orthoptera (da qual os gafanhotos fazem parte) na Área I pode estar relacionada com as constantes alterações provocadas pelos humanos no local, como as práticas agrícolas já citadas. Além disso, a Área I é considerada a mais antropizada, quando comparada a Área II que não sofre essas alterações ambientais. É interessante destacar que, de acordo com Nunes-Gutjahr e Braga (2010) algumas espécies de gafanhotos (Acrididae) são encontradas mais comumente em áreas antropizadas.

\subsection{Efeitos da temperatura e precipitação sobre os organismos da macrofauna edáfica}

A precipitação pluvial $(\mathrm{mm})$ nos meses estudados pode ter influenciada na abundância da macrofauna edáfica. Na tabela 2, consta a distribuição do número de indivíduos amostrados na Área I e Área II nos meses coletados.
Tabela 2. Número de indivíduos das ordens da macrofauna, e valores das variáveis ambientais verificados na Área I (APA do Buriti do Meio) e Área II (APA do Inhamum), Caxias, Maranhão. Fonte: Autor (2019).

\begin{tabular}{|c|c|c|c|c|c|}
\hline Meses & $\begin{array}{c}\text { Área } \\
\text { I }\end{array}$ & $\begin{array}{c}\text { Média } \\
\text { da } \\
\text { temp. } \\
\text { do } \\
\text { solo } \\
\left({ }^{\circ} \mathrm{C}\right) \\
\end{array}$ & $\begin{array}{c}\text { Área } \\
\text { II }\end{array}$ & $\begin{array}{c}\text { Média } \\
\text { da } \\
\text { temp. } \\
\text { do } \\
\text { solo } \\
\left({ }^{\circ} \mathrm{C}\right) \\
\end{array}$ & $\begin{array}{l}\text { Precipitação } \\
\text { (mm) }\end{array}$ \\
\hline Set/2018 & 1192 & 29 & 415 & 27 & 13,5 \\
\hline Out/2018 & 1046 & 32 & 1658 & 28 & 42,5 \\
\hline Fev/2019 & 884 & 28 & 625 & 27 & 182,2 \\
\hline Mar/2019 & 395 & 27 & 516 & 28 & 373,9 \\
\hline
\end{tabular}

Pode-se observar que na Área I ocorreu a maior abundância de indivíduos no período seco (setembro/2018 e outubro/2018), e houve redução no número de indivíduos no período chuvoso (fevereiro/2019 e março/2019), com ênfase para março/2019 com 395 indivíduos.. A temperatura do solo nesta área foi constante, com 28 e $27^{\circ} \mathrm{C}$ em fevereiro e março/2018, respectivamente. $\mathrm{Na}$ Área II, houve maior abundância de indivíduos no mês de outubro/2018, , o qual foi consideravelmente maior com relação ao demais meses.

Os resultados nos mostram que, possivelmente, as populações das ordens estudadas sofreram influência da variabilidade do regime pluviométrico. As variações de temperatura do solo e umidade características às estações do ano são fatores que apresentam uma influência direta na densidade faunística (SOARES; COSTA, 2001; FERNANDES et al., 2011). Pinheiro et al. (2014) avaliando a macrofauna edáfica na interface soloserapilheira em área de caatinga obteve resultado semelhante, onde a macrofauna foi mais abundante no período seco. Marques e Del-Claro (2010), também encontraram em um ambiente de cerrado, particularmente em vereda, as ordens Hymenoptera e Coleoptera como as mais abundantes na estação seca. No entanto, a maior abundância no período seco, pode estar relacionada aos indivíduos da ordem Hymenoptera, mais precisamente os formicídeos. Vasconcellos et al., (2013) associaram a alta abundância de formigas no período seco à maior disponibilidade e menor competição por alimento, e, segundo Collison et al., (2013), nessas condições a funcionalidade dos macroinvertebrados pode ser reduzida ocorrendo uma mudança na comunidade para espécies mais bem adaptadas a baixa umidade.

Segundo Thomas (2017), no período seco ocorre maior acúmulo de serapilheira no solo, já que neste período, a vegetação perde grande parte do seu dossel. A serapilheira acumulada fornece microclima favorável às espécies hipogéicas, que são espécies que forrageiam no interior do solo (SILVA et al., 2015). Assim, equilibra a temperatura do solo e propicia meios de nidificação e forrageamento para as espécies de formigas que circulam nele (ROCHA, 2012). 


\section{3. Índice de Riqueza de Margalef (I), Diversidade de Shannon (H') e Equabilidade de Pielou (e)}

A riqueza, verificada através do índice de Margalef (I), a diversidade de Shannon (H') e equabilidade de Pielou (e) das ordens da macrofauna encontradas na Área I e Área II, estão representadas na Tabela 3 .

Tabela 3. Índices de riqueza de Margalef (I), diversidade de Shannon-Wiener ( $\left.H^{\prime}\right)$ e equabilidade de Pielou (e) na APA do Buriti do Meio (Área I) e APA do Inhamum (Área II), Caxias, Maranhão. Fonte: Autor (2019).

\begin{tabular}{cccc}
\hline $\begin{array}{c}\text { Áreas } \\
\text { de } \\
\text { Estudo }\end{array}$ & $\begin{array}{c}\text { Índice de } \\
\text { Riqueza de } \\
\text { Margalef }(\mathbf{I})\end{array}$ & $\begin{array}{c}\text { Índice de } \\
\text { Shannon- } \\
\text { Wiener (H') }\end{array}$ & $\begin{array}{c}\text { Índice } \\
\text { Equabilidade } \\
\text { de Pielou } \\
(\boldsymbol{e})\end{array}$ \\
\hline Área I & 1,32 & 1,38 & 0,63 \\
Área II & 1,66 & 1,42 & 0,68 \\
\hline
\end{tabular}

De modo geral, foi observado que na Área II houve maior de riqueza, diversidade e equabilidade. Este resultado pode estar diretamente relacionado com a maior estabilidade do ambiente na Área II em relação à Área I, que se encontra em estágio avançado de sucessão secundária, pois o ambiente constantemente sofre processos de queimadas pelos agricultores, causando desequilíbrio na fauna e na flora. Em geral, áreas conservadas, estáveis ou com alto grau de recuperação, apresentam valores altos de diversidade (Shannon) e equabilidadede (Pielou) dos invertebrados edáficos devido à melhor estabilidade do sistema (NUNES et al., 2008; HOFFAMANN et al., 2009). Silva et al. (2014) afirmam que, o manejo adotado na área influencia diretamente a diversidade de espécies da fauna edáfica por ocasionar alterações físicas, químicas e biológicas no solo, considerando que, quanto maior a diversidade, melhores são as condições ecológicas da área. Além disso, a degradação dos ecossistemas altera a distribuição da fauna edáfica por limitar ou extinguir a disponibilidade de recurso alimentar, modificando as interações ecológicas e inviabilizando a sustentabilidade dos sistemas de produção (GOMIDE et al., 2011).

Os índices de diversidade Shannon (H') e equabilidade de Pielou ( $e$ ) em função dos meses coletados na Área I e Área II estão representados na Tabela 4. Para a Área I, observou-se que o maior índice de diversidade e equabilidade foi o mês de março/2019 e o menor foi o mês de setembro/2018.

Tabela 4. Índices de diversidade (Shannon-Wiener H') $e$ equabilidade (Pielou) entre os meses coletados na APA do Buriti do Meio (Área I) e APA do Inhamum (Área II), Caxias, Maranhão. Fonte: Autor (2019).

\begin{tabular}{cccc}
$\begin{array}{c}\text { Áreas de } \\
\text { Estudo }\end{array}$ & Mês/ano & $\begin{array}{c}\text { Índice de Shannon- } \\
\text { Wiener }\left(\mathbf{H}^{\prime}\right)\end{array}$ & $\begin{array}{c}\text { Índice de } \\
\text { Pielou }(\boldsymbol{e})\end{array}$ \\
\hline APA do & Set/2018 & 0,30 & 0,14 \\
Buriti do & Out/2018 & 0,49 & 0,22 \\
Meio & Fev/2019 & 1,22 & 0,59 \\
& Mar/2019 & 1,41 & 0,64 \\
\hline
\end{tabular}

\begin{tabular}{llll}
\hline & Set/2018 & 0,38 & 0,19 \\
APA do & Out/2018 & 0,33 & 0,17 \\
Inhamum & Fev/2019 & 1,26 & 0,58 \\
& Mar/2019 & 1,27 & 0,58 \\
\hline
\end{tabular}

Para a Área II, os meses de fevereiro/2019 e março/2019 foram os meses com maiores índice de diversidade e equabilidade, e o menor foi em outubro/2018. Essa variação da diversidade desses organismos edáficos é utilizado também como indicadora da resiliência do solo. Existe uma tendência de que os organismos edáficos respondam de maneira diferenciada aos manejos utilizados no solo, e a diversidade de espécies esteja diretamente ligada ao regime hídrico e clima de uma região, sendo assim um indicador biológico sensível (PELOSI; RÖMBKE, 2016).

No geral, foi observado que em ambas as áreas houve menor diversidade e equabilidade para o período seco (setembro e outubro). Tal acontecimento pode estar condicionado à presença de alguns grupos mais adaptados, que se tornam consequentemente mais abundantes, como é o caso dos indivíduos da ordem Hymenoptera. Segundo Bianchi et al., (2017), essa predominância do grupo, sobretudo dos formcídeos, causou as diferenças nos índices de diversidade de Shannon e de equabilidadede Pielou. A relação entre densidade de indivíduos e riqueza de grupos expressa por esses índices evidencia que no período chuvoso as áreas estavam representadas por uma comunidade mais diversa, na qual os grupos ocorreram de forma mais representativa e melhor distribuída, embora isso não signifique uma maior abundância total. Além disso, segundo Baretta et al. (2003), a perda de diversidade ocorre devido à presença de espécies dominantes, favorecidas pelo ambiente da parcela ou pelo fornecimento de um único substrato alimentar.

\section{CONSIDERAÇÕES FINAIS}

$\checkmark \quad$ Nas duas áreas estudadas foi encontrado um total de 15 ordens taxonômicas durante os quatro meses de coletas;

$\checkmark \quad$ A ordem Hymenoptera, nas duas áreas, foi considerada superdominante, superabundante, superfrequente e constante;

$\checkmark \quad$ A precipitação puvial pode interferer diretamente na abundância de indivíduos da macrofauna edáfica;

$\checkmark \quad \mathrm{O}$ ambiente da Área II apresentou maior riqueza, diversidade e equabilidade de grupos taxonômicos, de acordo com os índices de Margalef, Shannon e Pielou;

$\checkmark \quad$ O trabalho relevou que as práticas de manejo de agricultura interferem no comportamento da fauna do solo, indicando que a mesma é mais abundante em ambientes mais conservados, confirmando que os organismos edáficos são bons bioindicadores da qualidade do solo;

$\checkmark \quad$ A realização deste trabalho é o ponto de partida para a elaboração dos planos de manejo e conservação; é também uma importante ferramenta para o monitoramento ambiental. As informações obtidas a partir destes estudos são utilizadas para subsidiar o conhecimento da biodiversidade que ocorre nas áreas, bem como a elaboração e análise de pesquisas científicas e relatórios de impacto ambiental. 


\section{REFERÊNCIAS}

AQUINO, A.M. et al. População de minhocas em sistemas agroflorestais com café convencional e orgânico. Ciência e agrotecnologia, v. 32, n. 4, 1184-1188, 2008.

ARAÚJO, C.C. et al. Comparação da abundância de invertebrados de solo por meio da estimação intervalar encontrados em diferentes ambientes na cidade de Ituiutaba - MG. Bioscience Journal, v. 26, n. 5, 817-823, 2010.

ARAÚJO, F. A. S. A. Geomorfologia aplicada à fragilidade e ao zoneamento ambiental de Caxias/MA. Presidente Prudente, 2012. 185f. Tese (Doutorado em Geografia). Programa de Pós-Graduação em Geografia, Universidade Estadual Paulista Júlio de Mesquita Filho, Presidente Prudente-SP, 2012.

ARAUJO, K. D. Analise da vegetacao e organismos edaficos em areas de caating a sob pastejo e aspectos socioeconomicos e ambientes de Sao Joao do Cariri - PB. Campina Grande, 2010. 166f. Tese (Doutorado em Recursos Naturais). Programa de Pós-Graduação em Recursos Naturais, Universidade Federal de Campina Grande, Campina Grande-PB, 2010.

AUDINO, L. D. et al. Identificação dos coleópteros (insecta: Coleoptera) das regiões de Palmas (Município de Bagé) e Santa Barbinha (Município de Caçapava do Sul, RS). Bagé, RS: Embrapa Pecuária do Sul, 2007. 92p.

BARETTA, D. et al. Fauna edáfica avaliada por armadilhas de catação manual afetada pelo manejo do solo na região oeste catarinense. Revista Ciência Agroveterinárias, v. 2, n. 2, $97-$ 106, 2003.

BARETTA, D. et al. Efeito do cultivo do solo sobre a diversidade da fauna edáfica no planalto sul catarinense. Revista de Ciências Agroveterinárias, v. 5, n. 2, 108-117, 2006.

BARETTA, D.; BROWN, G.G.; CARDOSO, E.J.B.N. Potencial da macrofauna e outras variáveis edáficas como indicadores de qualidade do solo em áreas com Araucariaangustifólia. Acta Zoológica Mexicana, v. 2, n. 2 135-150, 2010.

BIANCHI, M.D.O. et al. Macrofauna edáfica como indicadora em revegetação com leguminosas arbóreas. Floresta $e$ Ambiente, v. 24, 2017.

BUSSINGER, P. D. Efeitos de Diferentes usos do Solo no Cerrado sobre a Composição da Fauna Edáfica. Brasília, 2018. 119f. Tese (Doutorado em Ciências Florestais) Programa de Pós-Graduação em Ciências Florestais, Universidade de Brasília. Brasília-DF, 2018.

BODENHEIMER, F. S. Precis d'écologie animale. Paris, França: Payot, 1955. 315p.

CARRILLO, Y. et al. Soil fauna alter the effects of litter composition on nitrogen mineral soil. Soil Biology and Biochemistry, v. 43, 1440-1449, 2011.
COLLISON, E.J.; RIUTTA, T.; SLADE，E.M. Macrofauna assemblage composition and soil moisture interact to affect soil ecosystem functions. Acta Oecologica, v. 27, n. 47, 3036,2013

FERNANDES, M.M. et al. Influência de diferentes coberturas florestais na fauna do solo na Flona Mário Xavier, no município de Seropédica, RJ. Floresta, v.41, n.3, 533-540, 2011.

GESTEL, C.A.M.; KRIDENIER, M.; BERG, M.P. Suitability of wheat straw decomposition, cotton strip degradation and bait-lamina feeding tests to determine soil invertebrate activity. Biol Fertil Soils, v. 37, 115-123, 2003.

GOMIDE， P.H.O.; SILVA， M.L.N.; SOARES， C.R.F.S Atributosfísicos, químicos e biológicos do solo em ambientes de voçorocas no município de Lavras - MG. Revista Brasileira de Ciência do Solo, v. 35, n. 2, 567-577, 2011.

GREGORY D, GIRIBERT G. Evolutionary biology of centipedes (Myriapoda: Chilopoda). AnnuRev Entomol, v.52, 151-170, 2007.

HOFFMANN, R.B. et al. Diversidade da mesofauna edáfica como bioindicadora para o manejo do solo em Areia, Paraíba, Brasil. Revista Caatinga, v. 22, n. 3, 117-121, 2009.

JACOBS, L. E. et al. Diversidade da fauna edáfica em campo nativo, cultura de cobertura milho + feijão de porco sob plantio direto e solo descoberto. In: XXXI CONGRESSO BRASILEIRO DE CIÊNCIA DO SOLO, 4, 2007, Gramado. Anais... Gramado: SBCS, p. 55-62, 2007.

KORASAKI, V. et al. Macrofauna. Conhecendo a vida do solo. Lavras, MG: Editora UFLA, 2017. 32p.

LOURENÇO, W. R.; EICKSTEDT, V. R. Escorpiões de Importância Médica. In: CARDOSO, J. L. C.; et al. Animais peçonhentos no Brasil: biologia, clínica e terapêutica dos Acidentes. São Paulo: Sarvier; Fapesp, 2009. p. 198-213.

MACHADO, D.L. et al. Fauna edáficanadinâmicasucessional da Mata AtlânticaemflorestaestacionalsemidecidualnaBacia do Rio Paraíba do Sul - RJ. Ciência Florestal, v. 25, n. 1, 91106, 2015.

MARQUES, G. D. V.; DELCLARO, K. Sazonalidade, abundância e biomassa de insetos de solo em uma reserva de Cerrado. Revista Brasileira de Zoociências, v. 12, n. 2, 141150,2010

MARINONE, R.C.; GANHO, N.G. A fauna de Coleoptera em áreas com diferentes condições florística no Parque Estadual de Vila Velha, Ponta Grossa, Paraná, Brasil. Abundância e riqueza das famílias capturadas através de armadilhas do solo. Revista Brasileira de Zoologia, v. 20, n. 4, 737-744, 2003 
MELO, F.V. et al. A importância da meso e macrofauna do solo na fertilidade e como biondicadores. ENCICLOPÉDIA BIOSFERA, Centro Científico Conhecer, v. 34 n. 1, 39-43,, 2009.

MONTES, M. L. et al. Zoneamento Geoambiental do Estado o Maranhão - Diretrizes Gerais Para a Ordenação Territorial. Ministério de Planejamento, Orçamento e Coordenação. Fundação Instituto Brasileiro de Geografia e Estatística. Salvador. p. 44, 1997. Disponível em: https://biblioteca.ibge.gov.br/visualizacao/livros/liv95885.p df. Acesso em: 12/02/2020.

MORAES, R. C. B. et al. Software para análisefaunística. In: $8^{\circ}$ Simpósio de controlebiológico. In: $8^{\circ}$ Siconbiol São Pedro, SP. Anais . v.1, n.1, 195, 2003.

NUNES, L.A.P.L.; ARAÚJO FILHO, J.A.; MENEZES, R.Í.Q. Recolonização da fauna edáfica em áreas de caatinga submetidas a queimadas. Caatinga, v. 21, n. 3, 214-220, 2008.

NUNES-GUTJAHR, A.L.; BRAGA, C.E. Similaridade entre amostras da acridofauna (Orthopera: Acrididae) em quatro áreas ao longo da estrada Santarém-Cuiabá (br-163), Pará, Brasil. Revista Nordestina de Zoologia, v. 4, n. 1, 118-130, 2010.

PELOSI, C.; RÖMBKE, J. Are Enchytraeidae (Oligochaeta, Annelida) good indicators of agricultural management practices. Soil Biology \& Biochemistry, v. 100, n, .255-263, 2016.

PINHEIRO, F.J. et al. Caracterização da macrofauna edáfica na interface solo serapilheira em uma área de caatinga do nordeste brasileiro. Enciclopédia biosfera, v. 10, n. 19, 2964, 2014.

RAFAEL, J. A. et al. Insetos do Brasil - Diversidade e taxonomia. Ribeirão Preto, SP: Holos Editora, 2012. 810p.

RENNER, L. M.; VELOSO, G.N.; SEGAT, J.C. A Importância da Faua do Solo. In Sul Brasil Rural. 2017. Disponível em: https://www.udesc.br/arquivos/ceo/id_cpmenu/1043/rural 205 15236482339303 1043.pdf. Acesso em: 12/02/2020.

ROCHA, W. O. Estudos da mirmecofauna aplicada na avaliação de áreas de garimpo de diamantes no município de Poxoréu, MT. Cuiabá, 2012. 53f. Dissertação (Mestrado em Ciências Florestais e Ambientais). Programa de Pós-Graduação em Ciências Florestais, Universidade Federal de Mato Grosso, Cuiabá-MT, 2012.

ROSA, M.G. et al. Macrofauna edáfica e atributos físicos e químicos em sistemas de uso do solo no Planalto Catarinense. Revista Brasileira de Ciência do Solo, v. 39, n. 6, 1544-1553, 2015.

ROSS, E.S. Origin, relationships and integumental anatomy of the insect arderEmbiidina. Embia, Part 1. Occasional Papers of the California Academy of Sciences 149: 1-53, 2000.
SANTOS, G. R. Dinâmica dos organismos edáficos e atividade microbiana, em áreas de Caatinga, Semiárido Alagoano. Maceió, 2014. 70f. Monografia (Graduação em Geografia). Instituto de Geografia, Desenvolvimento e Meio Ambiente, Universidade Federal de Alagoas, Maceió-AL, 2014.

SANTOS, G.R.; ARAUJO, K.D.; SILVA, F.G. Macrofauna edáfica na ecológica na Estação Ecológica Curral do Meio, Caatinga Alagoana. Revista de Geociências do Nordeste, v. 4, n. 2, 01-21, 2018.

SILVA, C.R.; SOUZA, K.B.; FURTADO, W.F. Avaliação do avanço da agricultura intensiva no Cerrado piauiense. Engevista, v. 16, n. 3, 432-439, 2014.

SILVA, R. R. et al. Grupos tróficos e guildas em formigas poneromorfas. In: DELABIE, J. H. C. et al. As formigas poneromorfas do Brasil. Ilhéus: Editus, 2015. p. 163-179.

SILVEIRA NETO, S. et al. Manual de ecologia dos insetos. São Paulo, SP: Ceres, 1976. 419p.

SOARES, M.I.J.; COSTA, E.C. Fauna do solo em áreas com Eucalyptus spp. e Pinus elliottii, Santa Maria, RS. Ciência Florestal, v. 11, n. 1, 29-43, 2001.

SOUZA, K. K. F. et al. Utilização de Formigas (Hymenoptera: Formicidae) como bioindicadoras em plantios de Pinus no Paraná. Revista Brasileira de Agropecuária Sustentável, v. 8, n. 1, 110-118, 2018.

THOMAS, S. E. O. Diversidade de formigas (Hymenoptera: Formicidae) em plantios de teca, pau-de-balsa e área nativa no bioma Cerrado. Cuiabá, 2017. 75f. Dissertação (Mestrado em Ciências Florestais). Programa de PósGraduação em Ciências Florestais, Universidade Federal de Mato Grosso, Cuiabá-MT, 2017.

TRIPLEHORN, C. A.; JONNSON, N. F. Estudo dos insetos. São Paulo, SP: Cengage Learning, 2011. 809p.

VASCONCELLOS R.L.F. et al. Soil macrofauna as an indicator of soil quality in a undisturbed ripariam forest and recovering sites of different ages. European Journal of Soil Biology, v. 58, n. 58, 105-112, 2013.

\section{AGRADECIMENTOS}

Agradecemosa Universidade Estadual do Maranhão-UEMA, pela concessão da bolsa do Programa Institucional de Bolsas de Iniciação Científica-PIBIC, , e a toda a Equipe do Laboratório da Fauna do Solo -LAFS por colaborar com a execução da pesquisa.

Recebido em: 13/02/2020

Aceito para publicação em: 22/03/2020 